\title{
Curiosa XIV
}

\author{
Georg Wilhelm Scheffers
}

Der Mathematiker Georg Wilhelm Scheffers wurde 1866 in Altendorf bei Holzminden geboren und starb 1945 in Berlin. Er studierte in Leipzig bei Klein, Engel und Lie. Bei Lie promovierte er 1890 auch; der Titel seiner Disseration lautet „Bestimmung einer Classe von Berührungstransformationsgruppen des dreifach ausgedehnten Raumes". Ebenfalls noch in Leipzig reichte er 1890 seine Habilitation ein. Im Jahr 1896 folgte er einem Ruf an die Technische Hochschule Darmstadt, von wo aus er 1907 an die Technische Hochschule in Charlottenburg, die Vorläuferin der heutigen TU Berlin, wechselte. Von 1911 bis 1912 war er dort Rektor, die Emeritierung erfolgte 1935. Für eine vollständige Biografie verweisen wir auf das ,MacTutor History of Mathematics“-Portal in
St. Andrews: www-history.mcs.st-andrews.ac.uk/Biographies/ Scheffers.html.

In der Mathematischen Fachbibliothek der TU Berlin haben die Mitarbeiter vor zwei Jahren Dokumente aus Scheffers' Nachlass gefunden. Scheffers schien eine gewisse Vorliebe für das leicht Schräge zu haben: Zwei Seiten aus der Gruppe „sonderbare Druckfehler" haben wir Ihnen in den Mitteilungen 22 (20/4), S. 98-99, gezeigt. Hier reproduzieren wir die Seite „XIV. Mittels einer Münze und eines Lineals die Mittelsenkrechte einer Strecke zu bestimmen“.

Wir danken Bärbel Erler und Jan Erdnüß (Mathematische Fachbibliothek der TU Berlin) sowie Irina Schwab (Universitätsarchiv der TU Berlin) für diesen großartigen Hinweis. (MJ)

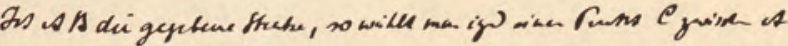

